Rev. SINAPSIS, Vol. 4, No 1, Junio 2014

\title{
Desarrollo de la competencia social en estudiantes del bachillerato Ecuatoriano desde la visión de la ciencia y la tecnología
}

\section{La competencia Social en Bachillerato Ecuatoriano}

\author{
Blanca Loor Lino. MSc. (1) \\ Ned Quevedo Arnaiz. Dr. C. (2) \\ Silvia Colunga Santos. Dra C. ${ }^{(3)}$ \\ (1)Unidad Educativa ITSUP, Portoviejo, Ecuador \\ (2)Universidad de Camagüey" Ignacio Agramonte Loynaz" Cuba. \\ ${ }^{(3)}$ Universidad de Camagüey" Ignacio Agramonte Loynaz" Cuba.
}

Contacto: blanloli@hotmail.com,

Receptado: 12/03/2014 Aceptado: 27/05/ 2014

\section{Resumen}

El presente trabajo es un acercamiento al desarrollo de la competencia social de los estudiantes del bachillerato desde la reflexión pedagógica actual mediada por los cambios que ocurren a diario y hacen que los procesos educativos constantemente se transformen. El objetivo es analizar el desarrollo de la competencia social, hablar eficazmente, en los estudiantes del bachillerato desde la perspectiva de usar la ciencia y la tecnología. Una mirada que necesita del análisis y la síntesis y del método sistémico entre otros para tener una aproximación en las soluciones que se esperan alcanzar con inmediatez en la juventud ecuatoriana. Se concluyó que si la comunicación se debilita en todos los contextos, la escuela no puede estar ajena a los cambios, ya que este problema repercute en el proceso educativo y la competencia social necesita ser tratada desde las interrelaciones sociales y la tecnología.

Palabras Clave: análisis y síntesis, ciencia y tecnología, comunicación, competencia social, habla eficaz, proceso educativo.

Development of social competence in high school students in Ecuador from the perspective of science and technology

\begin{abstract}
The present work is an approach to the developing of the social competence of high school students from the current educational thinking through changes occurring nowadays pedagogy and make educational processes constantly transformed. The objective is to analyze the development of social competence, the speaking effectively in high school students from the


Rev. SINAPSIS, Vol. 4, No 1, Junio 2014

perspective of using science and technology. A look that requires analysis and synthesis and the systemic approach including an approximation for the solutions to be achieved with immediacy in the Ecuadorian youth. It was concluded that if communication is weakened in all contexts, the school can not be oblivious to the changes, as this problem affects the educational process and social competence needs to be treated from social relationships and technology.

\begin{abstract}
The present work is an approach to the development of social competence in high school students from the current educational trends mediated by daily changes that provoke the constant transformation of the educational processes. The objective pursued is to analyze the development of social competence, speaking effectively, in high school students from the perspective of using science and technology. It is a look that requires analysis and synthesis and the systemic method included to reach at estimation for immediate solutions to be achieved in the Ecuadorian youth.
\end{abstract}

Keywords: analysis and synthesis, science and technology, communication, social skills, effective speaking, educational process

\title{
Introducción
}

La reflexión sobre los principios básicos de la educación se hace muy necesaria hoy, más aun con la aparición de los grandes modelos pedagógicos de desarrollo que revolucionan el conocimiento, el asesoramiento y la formación integral. La educación tiene como finalidad principal atender a la diversidad de sujetos que tiene la sociedad con todas sus necesidades reflejadas en los planteamientos actuales de inclusión, de allí que se deba insertar en los sistemas educativos con ritmo acelerado los descubrimientos derivados del desarrollo de la ciencia, con el fin de brindar una mejor educación

Así toda persona en cualquier posición en la que se encuentre en la sociedad, requiere de una buena dosis de capacidad de dirigirse hacia los demás para convencerlos, es una herramienta poderosísima de éxito y progreso. La satisfacción personal, comercial o social depende sobremanera de la capacidad que tiene una persona de comunicar claramente a los demás lo que siente, lo que desea y lo que cree. (Carnegie, 1968) 
Rev. SINAPSIS, Vol. 4, Nº 1, Junio 2014

A pesar de la declaración de la UNESCO (1994:3-4), que reconoce a “(...) La educación básica es más que un fin en sí misma. Es la base para un aprendizaje y un desarrollo humano permanentes...", no todos los países asumen este compromiso, ya que son otras las prioridades que se atienden como: pago de la deuda externa, desarrollo de planes económicos más ventajosos, proyectos con mayores ingresos, entre otras, lo cual puede causar retrocesos o estancamiento cultural, puesto que si no se beneficia la educación no se incrementa la cultura de los pueblos

Hoy en día es innegable que la ciencia, la tecnología han revolucionado la educación en general y las formas de comunicarse. Por ello, es necesario que como institución educativa ITSUP poner todo el interés ante la deficiente nivel de la habilidad hablar; que se manifiesta en la dificultad en el léxico que le imposibilita relacionarse con los demás, ya que no tienen fluidez verbal, ni dominio escénico. Por lo que se analizará en este trabajo desde la perspectiva crítica, constructiva el pro y el contra, el valor que adquiere el saber de estas, como condición indispensable para el desarrollo social de los pueblos.

Según Toffler (1994) expresa vivimos en una sociedad del conocimiento, caracterizada porque la base de la producción son los datos, las imágenes, los símbolos, los valores, la cultura, el lenguaje, la ciencia y la tecnología; porque lo más importante no es la infraestructura, los equipos, sino las capacidades de los individuos para obtener, crear, distribuir y aplicar creativa, responsable y críticamente los conocimientos en el contexto donde el ritmo de las innovaciones científicas crece aceleradamente y la tecnología de inmediato los va haciendo obsoletos.

Es por ello, que es necesario hacer una profunda reflexión desde los diferentes escenarios especialmente en la educación. Si se analizan estos modelos educativos se aprecia que estos entraron en crisis y las naciones más desarrolladas del planeta hacen esfuerzo desde diversos sectores para mejorar cuantitativamente los sistemas de formación tanto a niños, jóvenes y adultos.

El presente trabajo tiene como objetivo analizar el desarrollo de la Competencia Social: hablar eficazmente en los estudiantes del bachillerato desde la perspectiva de la ciencia y la tecnología 
Rev. SINAPSIS, Vol. 4, No 1, Junio 2014

ISSN 1390 - 7832

\section{Resultados}

Labatut (2004) La lingüística como otras ciencias del conocimiento humano presenta un debate entre el empirismo y el nativismo porque mientras los empiristas, conductistas Watson y Skinner. dicen: que los niños aprenden a hablar por imitación a los adultos, como sus padres ya sea por necesidades igualmente que desarrollan sus destrezas físicas y mentales y no por factores genéticos y de esta manera articulan y combinan sonidos. En cambio los Nativistas Gestall, Arnold y Gesell, rechazan estas teorías y dicen que el habla es un don biológico con el cual nacen todos los seres humanos y que la experiencia cognitiva es apenas un estímulo para el desarrollo posterior. Angulo (2007)

El lingüista norteamericano N. Chomsky padre de la Gramática Generativa plantea la teoría, que el niño tiene una programación genética para el aprendizaje de su lengua materna, desde el instante en que las normas para las declinaciones de las palabras y la construcción sintáctica de las mismas están ya programadas genéticamente en el cerebro y que lo único que hace falta es aprender a adaptar esos mecanismos gramaticales al léxico y las sintaxis del idioma materno.

Según Vigotsky, "El habla es, en primer lugar y ante todo, un instrumento de interacción social, un instrumento para opinar y comprender". (1982:17). Esta posición concuerda con la idea que defiende esta investigación. De acuerdo a la concepción histórico-cultural de la personalidad, se ha de reconocer que en el aprendizaje del individuo que se comunica ocurre como proceso en su interior pero también en la relación con otros como parte de la actividad social determinante.

Interesante resulta el análisis de estas teorías porque en todas de una forma u otra, se reflejan como son determinantes diversos factores sociales como por ejemplo: las relaciones sociales padres- hijos, procesos sociales como: los actos de imitación, el estímulo a la experiencia cognitiva, aprendizaje de la lengua materna.

En el Ecuador los problemas sociales son muchos ya sea por los malos gobiernos, crisis económica, la generalizada corrupción que se encuentra en todos los estratos sociales, la migración, las influencias externas, la educación de baja calidad con modelos pedagógicos descontextualizados que indudablemente se encuentran también en toda América Latina. 
Rev. SINAPSIS, Vol. 4, No 1, Junio 2014

ISSN $1390-7832$

Si se hace un análisis más general, cada día es acuciante la necesidad de tratar desde el aula los temas de comunicación, de conversación entre las personas ya que están ocurriendo disfunciones sociales que van a incidir en las relaciones sociales de los individuos limitando sus interacciones.

Por otro lado, baste con pensar y reflexionar seriamente sobre el tiempo empleado para abordar aspectos linguí́sticos, discursos, comunicación con procesos como; la oralidad, la lectura, la comprensión e interpretación principalmente académico-científico dentro de los procesos sociales.

El estudio de la competencia social hablar, que infortunadamente para muchos resulta desconocida, ya que creen erróneamente que con hablar, leer, escribir ya lo saben todo y que estos son procesos simples y sencillos que se pueden lograr en poco tiempo, constituye en el mundo contemporáneo en todos los contextos una necesidad social.

Chomsky (1975) al referirse a la Competencia hablar explica que se trata de un conocimiento intuitivo y práctico de un hablante ideal que lo capacita para producir oraciones y frases bien formadas

La competencia se diferencia de las habilidades necesarias para desempeñar una determinada tarea en un contexto social determinado, ya que una competencia es saber y saber hacer con otros y saber cuándo y por qué hay que utilizarlas.

Al enfocar la relación Ciencia, Tecnología y Sociedad desde la visión de Núñez Jover (1999) La ciencia exige que se le comprenda como proceso de investigación que permite obtener nuevos conocimientos, los que a su vez ofrecen posibilidades nuevas de manipulación de los fenómenos; es posible atender a sus impactos prácticos y productivos, caracterizándola como fuerza productiva que propicia la transformación del mundo y es fuente de riqueza; la ciencia también se presenta como una profesión debidamente institucionalizada portadora de su propia cultura y con funciones sociales bien identificadas.

La razón por la cual es posible apreciar tantas facetas diferentes de la ciencia es porque ella constituye un fenómeno complejo cuyas expresiones históricas han variado considerablemente. Por eso las definiciones de ciencia resultan escurridizas y a veces inalcanzables. 
Rev. SINAPSIS, Vol. 4, No 1, Junio 2014

El enfoque de la ciencia la obra de Núñez Jover se analiza como actividad y ofrece un excelente punto de partida para explorar sus relaciones con el marco cultural en que ella actúa. Examinar los rumbos de la cultura en la creatividad y los obstáculos a vencer es decisivo para entender las diferentes sociedades, sus tendencias de desarrollo, su vitalidad y capacidad de respuesta al reto que plantea el ambiente físico y social y las relaciones competitivas o hegemónicas que entre ellas se establecen. Para responder a esta expectativa, la cultura deberá pensarse como el proceso de asimilación, producción, difusión y asentamiento de ideas y valores en que se funda la sociedad; es el conjunto de representaciones colectivas, creencias, usos del lenguaje, difusión de tradiciones y estilos de pensamiento que articulan la conciencia social, es el ámbito en que se producen y reproducen nuestras formas de vida y nuestra ideología; vista así la cultura es un mecanismo de regulación social.

En lo que respecta a Tecnología, Marx (1973) descubre la actitud del hombre ante la naturaleza, el proceso directo de la producción de su vida y por tanto de las condiciones de su vida social y de las ideas y representaciones espirituales que de ella se derivan. La Tecnología designa la totalidad o una parte del conocimiento científico o empírico vinculado a las actividades industriales, los recursos

Entonces se puede decir que la ciencia y la tecnología juega un papel muy importante desde su invención y más aun en la primera década del siglo XXI ya que estas van a ritmo acelerado presentando una serie de transformaciones que a la vez generan una gran interrelación con la economía, la política, lo socio-cultural que se encuentran fundamentadas en políticas globales materiales.

Sin lugar a dudas, la competencia social hablar, necesita ser estudiada desde las interrelaciones de las ciencias y la tecnología, con todas las condicionantes sociales que en ella interactúan, pero esencialmente, como un proceso social cultural histórico.

En el lenguaje por su propia naturaleza se crean diferentes interrelaciones; sin embargo algunas de las ciencias que tributan con sus conocimientos al tema de investigación ubicado entre las Ciencias de la Pedagogía y Ciencias de la Educación son: la Historia , la Psicología Social, la Filosofía, la Sociología y la Psicopedagogía entre otras. 
Rev. SINAPSIS, Vol. 4, No 1, Junio 2014

ISSN $1390-7832$

Esta última, busca la educación en el ser humano trabajando el autoconocimiento ejecutando estrategias en la toma de decisiones y dotando de información que va a incidir en los comportamientos humanos.

Por otra parte, asumir el punto de vista científico, nos acerca el estudio de las competencias porque sobre ellas se plantean algunas interrogantes:

La 1era, es referida a las capacidades; ya que la competencia constituye una capacidad.

La 2da interrogante se refiere a la motivación de la competencia en las diferentes situaciones de aprendizaje y aquí se involucra (condiciones, situaciones, métodos, la forma de aprender).

La 3ra interrogante es el componente afectivo, social y personal de las competencias.

Rojas (1999. p. 28) define la competencia social como un constructo hipotético y teórico global multidimensional y amplio, mientras que las habilidades sociales pueden verse como parte del constructo de la competencia social.

Arnaiz (1995) considera que estas incluyen las conductas interpersonales lo que vale decir que la competencia social debe definirse como eficiencia y educación de los comportamientos del sujeto ya que esta forma parte de la conducta, de la adaptación del sujeto, incluyendo destrezas de funcionamiento independiente, desarrollo físico y de lenguaje.

En este análisis teórico, desde referentes científicos, se trasluce la interrelación entre la sociedad y la ciencia, promoviendo temas tales como personalidad y adaptación al medio, comportamiento de los sujetos de aprendizaje a través de la creación de capacidades, habilidades, y competencias.

En el contexto de la unidad educativa ITSUP por las dificultades antes señaladas en la competencia social hablar se demanda la creación de una atención pedagógica o didáctica dirigida a desarrollarla.

Se necesitan herramientas de trabajo en el proceso enseñanza-aprendizaje que sirvan para mediar el desempeño del profesor, del estudiante. Por ello es que se hace necesario el uso de alguna tecnología social. 
Rev. SINAPSIS, Vol. 4, Nº 1, Junio 2014

Importante es también definir ¿qué es una tecnología social, qué es una tecnología educativa? Porque para desarrollar la competencia social, hablar, se hace imprescindible el uso de una tecnología social, como son las normas y procedimientos para la producción de procesos sociales no necesariamente vinculados a la esfera productiva, sino a los servicios como son los educativos.

Cuando se habla de tecnología educativa: Se trata de un modo sistémico de concebir, aplicar y evaluar la totalidad del proceso educativo en función de unos objetivos precisos basados en investigaciones referentes a la instrucción y la comunicación humana, que utiliza un conjunto de medios humanos y materiales con el fin de dispensar una educación más eficaz.

La innovación es una combinación de necesidades sociales, de demandas sociales, con los medios científicos, tecnológicos para satisfacerla; incluye, por tanto, actividades científicas, tecnológicas, financieras y comerciales.

Se entiende por Innovación Tecnológica.-el proceso de crear capacidades que inciden en el incremento de la actividad inventiva en todos los campos, incentivando el ritmo de cambios tecnológicos, requisito básico para reanimar la vida económica social del mundo.

La popularización de la ciencia y tecnología debe simultáneamente ser potencial y vinculada a la afirmación de las capacidades propia de los países de América Latina y el Caribe y este proceso persigue que se constituyan en un componente central de la cultura, de la conciencia social y de la inteligencia.

Es necesario ampliar la utilización de la tecnología de información y de la comunicación en especial mediante la creación de redes. Así mismo se debe velar porque la utilización de estas no conduzcan a negar ni a limitar la riqueza de la cultura y de los diferentes medio de expresión. Que lejos de favorecer, su uso indiscriminado entorpezca el desarrollo social.

En muchos países la educación básica no está al alcance de todos y en otros no tiene la calidad necesaria para desarrollar el intelecto y las posibilidades de comunicarse. Por lo que se busca que las políticas educativas atiendan y creen conciencia sobre la adecuada educación básica que es necesaria para fortalecer los niveles superiores de la educación y de la enseñanza para alcanzar un desarrollo autónomo desde los procesos comunicativos y valorativos del sujeto. 
Rev. SINAPSIS, Vol. 4, Nº 1, Junio 2014

La Constitución del Ecuador consigna que la actividad educativa se desarrolla atendiendo a los principios generales que son los fundamentos filosóficos, conceptuales y constitucionales que sustentan, definen y rigen las decisiones y actividades en el ámbito educativo. Por tanto, la educación es un derecho humano fundamental y deber ineludible e inaccesible del estado. Esto la articula a los instrumentos de transformación de la sociedad porque la educación se constituye un instrumento de transformación a la convivencia del país, de los proyectos de vida y de la libertad de sus habitantes, pueblos, nacionalidades, reconoce a las y los seres humanos en particular a los niños, niñas, adolescentes, como cambio de procesos de aprendizajes.

La Ley Orgánica de Educación Intercultural pide: Investigar, sistematizar, llevar el registro lingüístico de los idiomas ancestrales de República del Ecuador y Formular las normas lingüísticas de los idiomas ancestrales de la República del Ecuador.

Toda esta normativa político- jurídica, en esta última dirección es vital en el contexto ecuatoriano, si tenemos en cuenta las diferencia dialectales que se evidencian de la siguiente manera: el hombre de la costa en la mayoría de sus palabras omite la s, el manaba por ejemplo suele confundir la tildación de las palabras, acortan o reducen consonantes y tienden más a lo diminutivo. A la vez se le hace difícil la distinción entre la b y la v, la z y la s, dimensionan o exageran la terminación ísimo. El montubio también reduce las palabras compa por compadre, y hace reducción para por pa.

En la Sierra varía de acuerdo a la clase social y al grupo que pertenece por ejemplo el dialecto de Imbabura es diferente, el dialecto en Cuenca de caracteriza por ser cantado, en Quito e Ibarra utilizan mucho el voseo, modismos; en cambio en Loja su entonación es neutral.

En la Amazonia el habla es parecido al dialecto andino ecuatoriano, ellos tutean y conjugan el verbo en usted. En cambio en Galápagos el dialecto es parecido al de la costa del Ecuador.

A toda esta problemática, de condicionantes sociales se añade la existencia de los medios que intervienen no siempre de forma adecuada en la conservación de la lengua autóctona. La sociedad en la actualidad se caracteriza por poseer flujos diversos y abundantes en información y comunicación. Un escenario poblado de imágenes, íconos, figuras que provienen de distintas latitudes. En un siglo se ha pasado del aparato radiofónico a la cinematografía en blanco y negro 
Rev. SINAPSIS, Vol. 4, No 1, Junio 2014

ISSN $1390-7832$

al color, a las noticias a nivel mundial, lamentablemente todo este desarrollo no tiene siempre un impacto social favorable.

El actual gobierno ha tomado a la educación como prioridad nacional entendiéndose no solo como servicio básico sino como un eje transversal de todas las políticas y sectores como requerimiento fundamental del desarrollo; el mismo que quiere hacer de esta una prioridad nacional para que sea entendida y asumida como tal contando con el ministerio del ramo y la interlocución de un consejo nacional de educación representado de todos los sectores y regiones del país.

La CEPAL (2001) reconocía la necesidad de elogiar y dar prioridad al gasto en salud y educación, por tal motivo los recursos para el financiamiento de políticas sociales creció notablemente hasta el punto que a finales de los años 90’s alcanzaron el nivel más elevado de la historia.

De esta manera el sistema nacional para brindar una protección integral a la comunidad con equidad social y atender la inclusión expide políticas que van a mejorar los procesos educativos y desarrollar las capacidades y potencialidades acorde a los nuevos retos que la sociedad impone, como son:

La investigación y el conocimiento científico promueven el fortalecimiento de la institucionalidad pública de la ciencia y tecnología, los proyectos y actividades de ciencia y tecnología en todos los niveles educativos e incorporación en las mallas curriculares de los contenidos vinculados. Se apoyan, además, las organizaciones dedicadas a la producción de conocimiento científico e innovación tecnológica, se promueve programas de extensión universitaria con enfoque intercultural, de género y generacional.

Otra política actual, promueve el acceso a la información y a las nuevas tecnologías de la información y comunicación para incorporar a la población a la sociedad de la información y fortalecer el ejercicio de la ciudadanía.

De materializarse todos estos objetivos se habrán, creado condiciones para la formación de competencias sociales, entre ellas, la de hablar. 
Rev. SINAPSIS, Vol. 4, No 1, Junio 2014

La interacción entre los seres humanos y sus obras han dado consecuencias de diferentes magnitudes. Ya que un proceso puede ser causa o consecuencia, según el momento que hagamos para definirlo y sintetizarlo.

Hoy el caudal de información se materializa mediante el internet o la red de redes en el proceso de la globalización o por medio de las nuevas tecnologías de principios que permiten hablar de un modo "protegido", pero a la vez en la familia ha desaparecido el diálogo.

Todas las personas hablan pero muy pocas lo hacen con eficacia, pues esta requiere de cultivo y de educación, de esta manera para una comunicación oral eficaz se necesita, un conocimiento de la lengua, de los procesos del habla eficaz, de las formas y de los géneros básicos, de la asertividad que genera la interacción, y el entendimiento de la realidad de los demás, entre otros. Si se toma en cuenta a la lengua como fenómeno social hay que resaltar que esta conlleva a un proceso de interacción y desarrollo, entonces la tarea comunicativa se convierte en la parte más elemental del proceso de enseñanza que busca desarrollar las habilidades comunicativas, generar competencias y brindar la oportunidad de actuar en cualquier momento.

La formación en la comunicación procura facilitar competencias que capaciten a los estudiantes para intervenir en diferentes grupos sociales de distinta afinidad e involucrarse, ser colaborativo y generar intercambios conversacionales. Pero la teoría no ha explicitado como ellas se particularizan en las asignaturas que en el bachillerato desarrollan habilidades comunicativas para que sirvan como competencia social para toda la vida, toda vez que en esa edad del estudiante hay carencias educativas que conspiran contra el hablar refinado. De allí que se descubre un vacío teórico en la forma en que se explica la generación de competencias sociales desde la interacción que en la adolescencia debe lograr el hablar eficaz y educado. En esta dirección serán las repercusiones que se esperan con la aplicación de la Estrategia educativa que se propone.

\section{Conclusiones}

El mundo se transforma a gran velocidad aunque no parece que los cambios sean positivos, porque la comunicación a pesar de esto se debilita, la escuela no puede estar ajena a los cambios, porque si se debilita la comunicación en la familia esto va a repercutir en el desempeño académico del estudiante y el proceso de conformación de la competencia social, hablar, ya que 
Rev. SINAPSIS, Vol. 4, No 1, Junio 2014

ISSN $1390-7832$

es un proceso social, que tiene que ser realizado teniendo en cuenta una profunda caracterización del contexto social dado.

Se necesita que la competencia social sea estudiada desde las interrelaciones de las ciencias y la tecnología, con todas las condicionantes sociales que en ella interactúan, pero esencialmente, como un proceso social cultural histórico. Por lo tanto se demanda en el contexto de la unidad educativa ITSUP por las dificultades existentes la creación e implementación de alguna propuesta didáctica que constituya en una tecnología social, viable y educativa.

\section{Bibliografía}

1. ANDREIE J 1979. Ciencia Y Progreso Social. Moscú Editorial Progreso.

2. ANGULO TAPIA, A. 2007. Influencia de la estimulación psicomotriz a través del juego en el desarrollo del lenguaje en niños y niñas de 3 a 5 años del preescolar ä del centro infantil Frau Klier de la ciudad de Quito durante el año lectivo 2006 - 2007. Tesis de Licenciatura en Psicología. Universidad Politécnica Salesiana de Quito.

3. BERNAL D 1986. Historia Social De La Ciencia. Habana Editora Ciencias Sociales, tomo 1 pagina 21-22.

4. CASANY Daniel 2000. La Antropología Digital. El Futuro De La Enseñanza.

5. CARNEGIE, D. 1968.Cómo Hablar en Público. Navarra

6. CASTRO, D. B. F (2001). Ciencia, Innovación y Futuro. Editorial Instituto Cubano del libro. Ediciones especiales. La Habana.

7. CULLER Jonathan 2000. Breve Introducción A La Teoría Literaria. Barcelona.

8. CHOMSKY, N. 2004. Estructuras Sintácticas Siglo 21. Editora S. A Décima sexta edición. Mexico.

9. FIGUEROA Castro 2000. Crítica Literaria Y Pedagogía En Escritores Y Profesores. I Foro Internacional. Bogotá.

10. GARCÍA Carmen 2000.Desarrollo de Habilidades Comunicativas.

11. LABATUT PORTILHO, E. M 2004. Aprendizaje universitario: un enfoque metacognitivo. Tesis doctoral. Universidad Complutense de Madrid. 
Rev. SINAPSIS, Vol. 4, No 1, Junio 2014

12. MARCHESS ULLASTRE, Álvaro 2005. La lectura como estrategia para el cambio educativo Revista De Educación. Edición especial.

13. MARX 1973. EL CAPITAL. Editora de ciencias sociales la Habana.

14. MORALEDA 1995. Comportamiento social en la infancia y la adolescencia. Valencia.

15. NÚÑEZ Jover, Jorge; (1999): La ciencia y la tecnología como procesos sociales. Editorial "Félix Varela", La Habana.

16. SAENZ. T · (1999) Ingenierización e Innovación Tecnológica en Tecnología y Sociedad CIEST. Editorial Felix Varela. La Habana.

17. SAEZ Antonio 2004. Compendio de Estudios Sociales sobre Ciencia y Tecnología para los doctorantes. Instituto José Martí.

18. SÁNCHEZ-Toledo, A.C.: Migración y desarrollo. El caso de América Latina, en $\begin{array}{llll}\text { Contribuciones a las Ciencias } & \text { Sociales, noviembre }\end{array}$ [www.eumed.net/rev/cccss/06/acst.htm] Consultado: 3 de mayo de 2013.

19. TOFFLER, A. 1994. La Guerra del Futuro. International Bestseller.

20. Tecnología Y Sociedad Colectiva De Autores, Editorial. Félix Varela 1999.

21. VAN Dijck 1995. Texto y Contexto Semánticos, Pragmáticos del Discurso. Madrid.

22. VIGOTSKY, L. 1982. Obras Completas; Vols I - IV. Moscú. Pedagogía. 